\title{
Editorial HMD 322 - Wertbeitrag Wissen
}

\author{
Stefan Reinheimer
}

(C) Springer Fachmedien Wiesbaden GmbH, ein Teil von Springer Nature 2018

\begin{abstract}
„Wissen ist Macht“ - so beginnt die Ankündigung dieser Ausgabe der HMD - Praxis der Wirtschaftsinformatik auf der Springer Web-Site (www.springer.com/hmd). Dieser These haben sich die Autoren des Heftes verschrieben. Nach dem Aufkommen des Knowledge Management im letzten Jahrhundert, das oftmals eher einem „Happy-Engineering-Ansatz" folgte und dabei nicht immer realitäts- und praxisorientiert eingeführt wurde, steht bei der aktuellen Welle von Wissensmanagementaktivitäten der unternehmerische Nutzen im Vordergrund - Technik und Technologie als Mittel zum Zweck anstatt als Selbstzweck.

In einer großen Bandbreite von Beiträgen wird das Thema von verschiedenen Seiten beleuchtet - aus akademischer und aus praktischer Sicht, aber immer erkenntnisorientiert für den geschäftlichen Alltag:

Nach einem Grundlagenbeitrag, der einen historischen und fachlichen Abriss der Entwicklung des Knowledge Management und seiner Bedeutung im Unternehmen gibt, liefert eine empirische Studie von Stellenausschreibungen Fakten über gesuchte Kompetenzen von Innovationsmanagern - einer speziellen Rolle im Wissensmanagement. Einen schönen Kontrast zu konzeptionell und technisch durchgestylten Ansätzen bietet der dritte Beitrag. Nachdem ich selbst ein mittelständischer Unternehmer bin, kann ich aus meinen persönlichen Erfahrungen und denen meiner Umgebung bestätigen, dass Bauchentscheidungen eine signifikante Rolle im Management spielen - und sich dadurch häufig auch gute von schlechten Unternehmen unterscheiden - trotz der umfangreichen Verfügbarkeit von Wissens- und Management-Systemen. Dieses Thema wird hier aufgegriffen.
\end{abstract}

S. Reinheimer $(\bowtie)$

Nürnberg, Deutschland

E-Mail: sr@bik.biz 
Moderne Technologie ist die Grundlage der nächsten beiden Beiträge: Wissensarbeit mit Social Media Plattformen und ein Praxisbeispiel zum Einsatz von Chatbots bei der Schweizer Post. Auch der nächste Artikel ist der Praxis entnommen - ein Projekt zur Abbildung von Beziehungswissen komplexer Produkte der Siemens AG mit Hilfe semantischer Netze. Eine Technologie der Zukunft!?

Wertenetze und subjektorientiertes Geschäftsprozessmanagement werden als methodische Grundlagen für die im folgenden Beitrag thematisierte Organisationsentwicklung herangezogen.

Unter dem Schlagwort „Gamification“ werden spielerische Ansätze in den geschäftlichen Alltag übernommen. In welcher Form dies zur Unterstützung des Informations- und Wissensaustauschs im Unternehmen eingesetzt werden kann, ist Gegenstand des achten Beitrages. Auch der darauffolgende Artikel beschäftigt sich mit der Berücksichtigung von Akzeptanzkriterien für Wissensmanagementsysteme und zeigt Ansätze zu nutzerorientierten Lösungen auf. Der abschließende Beitrag des Heftschwerpunktes befasst sich mit der Technologieauswahl zur Virtualisierung von Consultingleistungen.

Drei Außerhalbbeiträge sorgen dafür, dass das Heft nicht ausschließlich auf das gewählte Schwerpunktthema fokussiert, sondern die Breite der Wirtschaftsinformatik repräsentiert. Einem methodisch orientierten Beitrag, der ein Reifegradmodell für den 3D-Druck vorstellt, folgen zwei eher empirische Artikel: Im ersten werden mit Hilfe bereits etablierter Ansätze des Process-Minings Studienverläufe analysiert ein ungewöhnliches Einsatzfeld für diese Methode. Der andere Außerhalbbeitrag bereitet Umfrageergebnisse zum Status Quo der Business-Analyse in unserem Umfeld auf und vertieft das Thema in Form eines konkreten Beispiels.

Wie Sie es als Stammleser gewohnt sind, ergänzen zwei Buchbesprechungen die Fachbeiträge. Neben einer komprimierten, praxisnahen Anleitung zum effizienten Einsatz von Wissensmanagementsystem im Umfeld des Qualitätsmanagements habe ich diesmal einen Wirtschafts-Bestseller besprochen, den ich Ihnen nur wärmstens empfehlen kann.

Ich bin davon überzeugt, dass das vorliegende Heft interessante Perspektiven auf den wertschöpfenden Beitrag von Wissen im Unternehmen bietet - bemächtigen Sie sich dieses Wissens!

Viel Spaß beim Lesen!

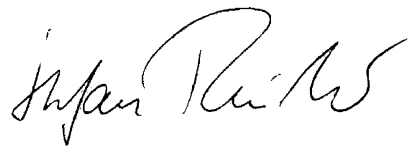

Stefan Reinheimer 